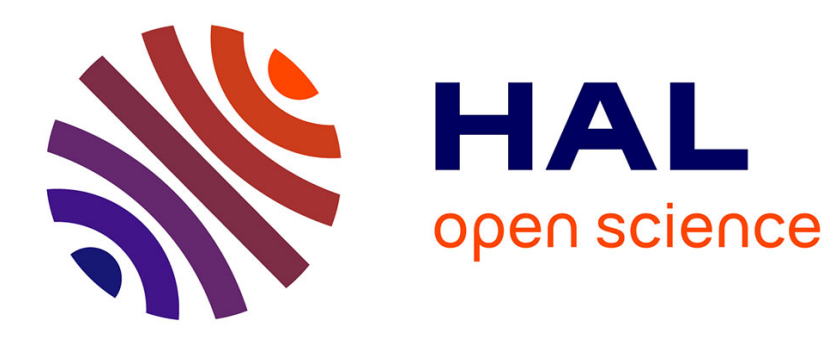

\title{
Paradoxes du travail social au sein des associations de lutte contre les violences conjugales Elisa Herman
}

\section{To cite this version:}

Elisa Herman. Paradoxes du travail social au sein des associations de lutte contre les violences conjugales . Informations sociales, 2012, 169. halshs-01674948

\section{HAL Id: halshs-01674948 \\ https://shs.hal.science/halshs-01674948}

Submitted on 3 Jan 2018

HAL is a multi-disciplinary open access archive for the deposit and dissemination of scientific research documents, whether they are published or not. The documents may come from teaching and research institutions in France or abroad, or from public or private research centers.
L'archive ouverte pluridisciplinaire HAL, est destinée au dépôt et à la diffusion de documents scientifiques de niveau recherche, publiés ou non, émanant des établissements d'enseignement et de recherche français ou étrangers, des laboratoires publics ou privés. 


\title{
PARADOXES DU TRAVAIL SOCIAL AU SEIN DES ASSOCIATIONS DE LUTTE CONTRE LES VIOLENCES CONJUGALES
}

\author{
Elisa Herman
}

CNAF | Informations sociales

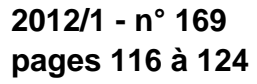

ISSN 0046-9459

Article disponible en ligne à l'adresse:

http://www.cairn.info/revue-informations-sociales-2012-1-page-116.htm

Pour citer cet article :

Herman Elisa, «Paradoxes du travail social au sein des associations de lutte contre les violences conjugales », Informations sociales, 2012/1 n¹69, p. 116-124.

Distribution électronique Cairn.info pour CNAF.

(c) CNAF. Tous droits réservés pour tous pays.

La reproduction ou représentation de cet article, notamment par photocopie, n'est autorisée que dans les limites des conditions générales d'utilisation du site ou, le cas échéant, des conditions générales de la licence souscrite par votre établissement. Toute autre reproduction ou représentation, en tout ou partie, sous quelque forme et de quelque manière que ce soit, est interdite sauf accord préalable et écrit de l'éditeur, en dehors des cas prévus par la législation en vigueur en France. II est précisé que son stockage dans une base de données est également interdit. 


\section{Paradoxes du travail social au sein des associations de lutte contre les violences conjugales}

Elisa Herman - sociologue

Dans les structures d'accueil de femmes victimes de violences conjugales, qu'elles furent les premières à créer, les associations féministes pratiquent un accompagnement spécifique. Il est contraint d'articuler la conscientisation féministe, qui fait des violences contre les femmes une question politique, et les logiques managériales imposées par les financeurs institutionnels, notamment l'activation pour le retour à l'emploi.

Le travail d'accompagnement des femmes victimes de violences conjugales, réalisé par des associations féministes qui les accueillent ou les hébergent, s'inscrit au croisement d'un héritage militant et d'une institutionnalisation dans le travail social. S'intéresser aux effets de ce travail social suppose alors d'être attentif à ces deux dimensions : observer, dans un premier temps, la dimension militante de cet accompagnement et, dans un second temps, les paradoxes rencontrés au niveau des logiques de réinsertion des femmes accueillies. Cet article aborde les effets sur les bénéficiaires de ce travail social à la lisière du militantisme, en proposant d'analyser, d'une part, les dynamiques de conscientisation qui émergent à certaines occasions et, d'autre part, les dynamiques de réinsertion des femmes accueillies conduisant les associations à composer entre les impératifs managériaux de l'institutionnalisation et les idéaux qu'elles défendent.

Qu'est-ce qu'un travail social féministe ?

Les bénéficiaires de cet accompagnement sont ainsi fréquemment issues des classes populaires et intermédiaires et ne représentent pas la totalité des femmes victimes de violences dans le couple (Jaspard et équipe Enveff, 2003). Ce public a donc des caractéristiques sociales assez similaires à 
celles des usagers du travail social classique et exclut, sauf exception, les femmes de milieu aisé, qui ont vraisemblablement les ressources financières et relationnelles nécessaires pour être soutenues (par des psychologues et des avocats en libéral), voire hébergées en dehors des réseaux associatifs (1). Néanmoins, pour les associations, les femmes de tous milieux sociaux devraient théoriquement pouvoir bénéficier de l'accompagnement spécifique mis en œuvre dans les structures d'écoute, d'accueil et d'hébergement. Ces associations, rassemblées au sein de la Fédération nationale solidarité femmes (FNSF), cherchent en effet à développer une spécificité dans leur pratique d'accompagnement, et à être reconnues à ce titre.

Cette spécificité, qu'on désigne ici comme un «travail social féministe » pour le décrire sociologiquement, a une histoire qui débute avec les «groupes femmes» ou "groupes de conscience» (2) nés dans les « années mouvement» (Picq, 1993 ; Mathieu, 2002). Ces groupes se sont transformés par l'institutionnalisation des associations, devenues progressivement auprès des victimes la principale composante active d'une politique publique de plus en plus affirmée. Jeannie, cofondatrice et coresponsable d'une structure à Toulouse, explique : «Ce sont les féministes qui ont dénoncé les violences comme un problème. Le féminisme des années 1970, son radicalisme, nous avons été nombreuses à le vivre dans ce que nous appelions des groupes de conscience ou des groupes de femmes. Ils étaient profondément politiques. C'était le mouvement antiautoritaire ou antipouvoir qui déconstruisait beaucoup de choses ». Elle poursuit en décrivant l'institutionnalisation devenue nécessaire : «On n'a plus compté seulement sur nos propres forces, car on pensait vraiment pouvoir proposer un autre système. On a voulu une reconnaissance des pouvoirs publics: être agréé en Centre d'bébergement et de réinsertion sociale (CHRS), adopter des conventions collectives ».

Ce travail social féministe s'appuie sur la politisation de la question des violences conjugales initiée par les mobilisations, politisation qui requalifie la question (Lagroye, 2003), en désignant certains actes dans le couple comme des violences intolérables, et subvertit collectivement différentes frontières sociales, en premier lieu celle séparant le public du privé. Ces mobilisations, complétées par les recherches et prises de positions académiques, construisent une cause publique, en prenant appui sur un cadre d'interprétation au cours d'opérations de cadrage successives, au sens donné par l'analyse des cadres (Goffman, 1991 ; Snow et al., 1986 ; Contamin, 2010). L'institutionnalisation (Lagroye et al., 2006; Lagroye et Offerlé, 2011) s'amorce ensuite par une professionnalisation et une dépendance à différents égards envers l'État, dans un processus qui met à l'épreuve la politisation de la question des violences contre les femmes. Le travail social féministe est donc une pratique hybride et non homogène, qui résulte de cette histoire et du processus, toujours en cours, d'institutionnalisation et de transformation de la politisation de cette cause publique. 


\section{Idéal féministe et injonction à l'autonomie économique}

Naissant de cette pratique d'accompagnement qui cherche à subvertir la frontière entre le militant et le professionnel, un autre effet notable de ce travail social féministe est le paradoxe dans lequel sont placées les salariées et les femmes accueillies; elles doivent composer avec une injonction à l'autonomie économique, les amenant à rechercher un emploi quel qu'il soit, de manière à pouvoir quitter les lieux d'hébergement ou le domicile conjugal.

Ces centres d'accueil et d'hébergement sont financés par des fonds publics dont les demandes et le pouvoir d'orientation du travail sont de plus en plus contraignants. Ils emploient des travailleuses sociales initialement formées à une pratique standardisée du travail social, c'est-à-dire très souvent individualisé, sous forme de contrat et d'objectifs et tourné vers la réinsertion sociale par le travail, dans une optique de réparation individuelle plutôt que de transformation des rapports sociaux. C'est pourquoi l'« auto-

$\boldsymbol{\zeta} \boldsymbol{V}_{(. . .)}$un autre effet notable de ce travail social féministe est le paradoxe dans lequel sont placées les salariées et les femmes accueillies (...). nomie » que doit acquérir la personne est une notion qui se trouve au centre du travail social actuel, présumant au départ qu'il existe une «dépendance» qui justifie l'intervention sociale. Cette notion, en partie issue du New Public Management et du principe d'activation, est très présente dans des dispositifs d'aide sociale tels que le Revenu de solidarité active (Duvoux, 2009).

L'observation des usages de la notion d'autonomie dans les structures dédiées aux victimes de violences conjugales montre qu'elle se décline sous trois formes repérables dans les pratiques d'accompagnement et les discours des salariées : autonomie administrative, psychique, économique. - L'autonomie administrative désigne l'ensemble des démarches à accomplir (relatives à un divorce, un titre de séjour, des prestations sociales, une procédure judiciaire, etc.) qui sont censées révéler l'autonomie de la personne (par sa capacité à les accomplir) et réaliser son autonomie quand elles aboutissent (par l'obtention d'un titre de séjour, d'un divorce, etc.). - L'autonomie psychique répond au concept «d'emprise» mobilisé pour expliquer la difficulté éprouvée par les femmes victimes à quitter un conjoint violent. Elle se comprend aussi comme une hybridation de concepts féministes et psychologiques pour penser les violences conjugales dans un contexte de psychologisation du travail social.

- L'autonomie économique désigne la capacité de trouver des revenus autres que les prestations sociales (rarement acceptées comme suffisantes par les bailleurs) pour assumer les charges matérielles quotidiennes et, ainsi, pouvoir quitter soit le centre d'hébergement, soit le foyer partagé avec le conjoint violent (3). Cela implique pour les femmes accueillies de se conformer aux critères « d'employabilité » sans avoir le temps d'effectuer 


\section{Encadré méthodologique}

Cette recherche est le fruit d'une thèse de sociologie en cours de finalisation intitulée provisoirement "Lutter contre les violences conjugales. Sociogenèse et ethnographie d'une politique publique ».

L'enquête repose sur une ethnographie, conduite dans un premier temps auprès d'associations féministes rassemblées au sein de la Fédération nationale solidarité femmes (FNSF), au fil de deux années d'observations, dont plus de six mois en observation participante dans un lieu d'accueil et d'hébergement. Ces associations hébergent et accueillent depuis le milieu des années 1970 des femmes victimes de violences avec leurs enfants. Elles se sont progressivement institutionnalisées au sein du travail social, en participant à l'élaboration de l'actuelle politique publique de lutte contre les violences envers les femmes.

Les relations interinstitutionnelles nouées par les associations avec la police, la justice, I'action sociale et les professionnels de la santé ont fait l'objet d'un second temps de l'enquête. La méthode ethnographique s'est déroulée entre 2006 et 2010, principalement en région parisienne, et a emprunté aux diverses ressources mobilisables. D'une part, près de 120 entretiens ont été réalisés, sous forme de récits de vie ou de type semi-directif selon les acteurs, auprès des salariées, des femmes accueillies, des militantes féministes, des professionnels extérieurs (police, justice, action sociale, santé, personnel politique...) et des universitaires. D'autre part, un travail documentaire sur l'ensemble des écrits collectés dans l'enquête a été conduit. Enfin, l'enquête repose principalement sur les observations directes consignées dans un journal de terrain durant deux ans - observations qui furent participantes durant une période.

de réelles formations, dans une période où les salariées leur préconisent au contraire de faire une pause et de prendre un temps de réflexion pour être ensuite capables de «reprendre main sur leur vie».

Confrontées à l'injonction à l'autonomie économique, les salariées sont prises dans de fortes contradictions entre la logique féministe et le contexte institutionnel qui les lie à des financeurs publics. Ces derniers, souhaitant rentabiliser les places d'hébergement financées, incitent les femmes à quitter au plus vite les espaces d'accueil, au prix de leur déqualification et de leur cantonnement dans des emplois précaires et très féminisés, reconduisant ainsi la division sexuelle du travail ordinaire. Car si l'indépendance financière des femmes fait partie de l'idéologie féministe, en revanche, l'indépendance conquise au prix d'autres formes d'oppression, par exemple par le travail salarié, devient problématique.

Cette situation d'entre-deux - agir professionnellement pour remplir un objectif militant (appliquer les valeurs féministes) dans un espace institutionnel global - produit donc des effets concrets sur les pratiques d'accompagnement, sur les salariées ainsi que sur le public reçu. La démarche féministe de subversion de frontière rencontre en effet le paradoxe suivant: si «l'autonomie » est une norme sociale qui trace une frontière entre les autonomes et les assistées, force est de constater que les femmes peuvent être dominées, qu'elles soient autonomes économiquement ou assistées. Le dépassement de cette frontière devient alors une question pour les militantes, davantage au niveau des organisations que des salariées (FNSF, 2006). 


\section{Conscientisation et conversions militantes}

Nous avons choisi d'exposer ici une pratique à la marge du quotidien des relations ordinaires entre femmes victimes de violences et travailleuses sociales en association féministe. Si elle est à la marge parce qu'elle n'est pas fréquente, cette pratique est finalement centrale par l'objectif militant qu'elle poursuit. Il s'agit de l'organisation, par deux associations, d'une campagne d'information aboutissant à une conférence publique au conseil régional d'Île-de-France pour dénoncer et lutter contre les discriminations freinant «l'accès au logement des femmes victimes de violences conjugales ». À la frontière entre travail social et militantisme, cette opération a impliqué un groupe de femmes bénéficiaires des associations et volontaires dans la préparation de la conférence, à raison d'au moins deux soirées par mois durant près de six mois. Animé par deux consultantes extérieures, ce projet s'est appuyé sur une méthode de "concertation créatrice » (4) visant à faire émerger une parole collective, restaurer l'estime de soi et permettre une transmission et une mise à jour des savoirs. Cette pratique d'accompagnement quasi militante, que nous avons suivie de bout en bout lors de l'enquête ethnographique (5), présente l'intérêt sociologique de rendre plus visible la spécificité de cet accompagnement, en pointant un effet rare et crucial chez les femmes accompagnées : celui de la conscientisation, de la découverte du féminisme, voire de la conversion au militantisme. Cette conscientisation se fonde sur une politisation de la question des violences conjugales, politisation qui est au cœur de la démarche féministe et qui s'est maintenue, en se transformant plus ou moins au fil du processus d'institutionnalisation entamé au début des années 1980.

\section{Une campagne contre les discriminations au logement envers les femmes victimes de violences}

Les femmes participant à la campagne ont dénoncé une stigmatisation et des représentations péjoratives à leur égard, amenant les bailleurs du parc social local à refuser leurs candidatures ou à traiter très lentement leurs demandes, alors que ces femmes, comme les associations (via une expertise professionnelle), considèrent qu'elles sont «prêtes au relogement» (6). Deux intervenantes, une sociologue et une urbaniste, ont été missionnées pour préparer le colloque et mener à bien trois objectifs :

- recenser, grâce aux femmes concernées, les vécus et les expériences des échanges avec les bailleurs du parc social ;

- initier un débat où se structurent les argumentaires pour convaincre les bailleurs et, au-delà, les pouvoirs publics ;

- permettre une initiation à la prise de parole publique pour que ces femmes, souvent peu diplômées et originaires des classes populaires, ne soient pas défavorisées lors du colloque (7). 
Cette initiative visait à améliorer la relation entre les femmes et les bailleurs, à transformer les représentations des bailleurs et à rendre plus rapide et plus importante l'attribution de logements sociaux. Des résultats significatifs ont été obtenus. Une promesse faite lors du colloque a été réalisée peu après : un petit nombre mensuel de logements dont l'attribution dépend de la Région a été en quelque sorte "préempté » et leur attribution confiée à la FNSF. Celle-ci peut alors faire circuler des annonces, en interne dans son réseau d'associations en France, pour que seules des femmes victimes de violences hébergées se portent candidates.

Le répertoire d'action militant mobilisé pour cet événement convoque la mémoire des activités féministes inventives des années 1970. Renouvelant les "groupes femmes ", utilisant des techniques sportives et de méditation (yoga, tai-chi, etc.) pour s'affirmer publiquement, et mobilisant le registre de l'éducation populaire, l'inscription dans une démarche féministe s'élabore par le répertoire d'action comme par les revendications. Dans le même temps, le registre du travail social classique est également sollicité, et la notion d'autonomie (économique et/ou psychique) soutient l'argumentaire. Le volet «psychique » de l'autonomie peut ainsi être travaillé par certaines professionnelles, avec l'objectif que les femmes accompagnées se défassent de l'emprise du conjoint mais aussi de l'emprise d'une conception traditionnelle des rôles sexués qui entrave l'égalité et l'épanouissement personnel.

Certaines femmes accompagnées, notamment celles qui ont participé à cette campagne, ont ainsi adopté une pratique militante nouvelle et sont devenues féministes, au sens où s'est opérée une conversion de leur regard sur leur vécu. Les rapports sociaux de sexe leur apparaissent alors comme une dimension explicative centrale pour comprendre leur trajectoire (professionnelle, matrimoniale, parentale...), et notamment les violences subies. Cela ne signifie pas nécessairement qu'elles vont par la suite adhérer à une association (quoique deux femmes ayant participé à ce groupe ont ensuite rejoint le conseil d'administration de la structure où elles avaient été hébergées), mais qu'elles se sentent désormais proches de la tradition féministe de lutte pour une égalisation des droits.

L'accompagnement féministe, en proposant des initiatives de participation citoyenne telles que ce type de campagne de sensibilisation, permet de réaffirmer un pouvoir de mobilisation pour les associations concernées (aux fins de négocier publiquement l'organisation et la mise en œuvre de la politique publique). Dans le même temps, leur participation peut être vécue par les femmes accompagnées comme une expérience positive d'activité « quasi politique », dans le cadre de laquelle coexistent affirmation de soi et découverte de l'action collective et s'amorce une politisation des 
individus. Les effets de l'accompagnement social par les associations féministes sont donc multiples et paradoxaux pour le public des femmes victimes de violences conjugales : ils vont de la conscientisation permettant de s'approprier la notion d'égalité des sexes, voire un répertoire d'action militant, jusqu'à une réinsertion qui, menée dans l'esprit d'activation caractéristique des politiques sociales actuelles, reconduit la division sexuelle du travail préjudiciable aux femmes.

\section{Notes}

1 - Les salariées des associations font ces observations à partir des relations tissées avec des avocats et praticiens de santé libéraux. En l'absence d'enquête dédiée à cette question, il n'est pas possible de statuer avec exactitude sur les recours et réseaux d'aide dont disposent les femmes de milieu favorisé qui subissent des violences. Néanmoins, certaines d'entre elles sont en contact avec des associations, ou hébergées par les services d'urgence, et elles sont nombreuses à contacter les permanences d'écoute téléphonique nationales et locales.

2 - La démarche de "conscientisation " que s'est approprié le mouvement féministe dans les années 1970 s'inspire de la théorie de la "pédagogie des opprimés ", travaillée par Paolo Freire au Brésil. Son point de départ fut une pratique d'alphabétisation qui devint le support d'un projet de transformation sociale par une libération des personnes par elles-mêmes, grâce à une revalorisation des savoirs "profanes " et à la prise de conscience des rapports sociaux. Cette référence, fréquemment citée par les actrices du Mouvement de libération des femmes (MLF) dans les années 1970, est encore aujourd'hui souvent convoquée.

3 - La possibilité pour les femmes victimes de violences de conserver le logement, par une mesure judiciaire par exemple, est encore peu mise en œuvre pour différentes raisons, notamment le danger auquel les femmes et leurs enfants restent exposés en continuant de vivre dans les mêmes lieux.

4 - Pour la présentation de leur démarche, voir Poggi, 2010, p. 83-89.

5 - Cet article s'appuie sur les entretiens et le journal de terrain décrivant ce projet particulier et sur une synthèse donnant la parole aux différents acteurs, L'accès au logement des femmes victimes de violences conjugales. Conférence du 11 octobre 2007, éditée par les associations L’Escale et Le Relais de Sénart, 2007, et disponible sur http://www.lescale.asso.fr/pages/partenariat-projets.htm. Ainsi que sur les textes élaborés par les femmes ayant participé au projet et publiés dans «Les Escaladeuses », in Francequin G. (dir.), 2010.

6 - Cette campagne fait également suite à la publication d'un rapport national dénonçant ces difficultés : Korsak B. (de) et Léger A.-M., Rapport sur l'hébergement et le relogement des femmes victimes de violences, Paris, La Documentation française, 2006, disponible sur http://www.ladocumentationfrancaise.fr/rapports-publics/064000268/index.shtm/

7 - D'autres initiatives portant sur d'autres thèmes ont été conduites par la suite par le même procédé, avec un groupe fluctuant mais conservant certains de ses membres. 


\section{Bibliographie}

- Aït-Aoudia M. et al., 2011, "Indicateurs et vecteurs de la politisation des individus : les vertus heuristiques du croisement des regards", Critique internationale, 1/2011, $\mathrm{n}^{\circ}$ 50, p. 9-20.

- Bessin M., Bidart C. et Grossetti M. (dir.), 2010, Bifurcations. Les sciences sociales face aux ruptures et à l'événement, Paris, La Découverte, coll. "Recherches».

- Contamin J.-G., 2010, "Cadrage et lutte de sens", in Fillieule O., Agrikolanski E. et Sommier I., Penser les mouvements sociaux. Conflits sociaux et contestations dans les sociétés contemporaines, Paris, La Découverte, coll. «Recherches », p. 55-75.

- Duvoux N., 2009, L'autonomie des assistés. Sociologie des politiques d'insertion, Paris, Presses universitaires de France (Puf), coll. " Le lien social ».

- Francequin G. (dir.), 2010, Tu me fais peur quand tu cries ! Sortir des violences conjugales, Toulouse, Érès, coll. "Sociologie clinique ».

- Freire P., 1974, Pédagogie des opprimés, Paris, Maspero, coll. «Petite collection Maspero ».

- Goffman E., 1991, Les cadres de l'expérience, Paris, Les Éditions de Minuit

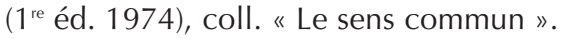

- Herman E., 2011a, " Droits des femmes migrantes et traitement social des violences conjugales », Hommes et migrations, $\mathrm{n}^{\circ} 1290$, mars-avril, p. 108$117 ; 2011$ b, "Idéal féministe et injonction à l'autonomie économique. Le travail social des associations luttant contre les violences conjugales", Pensée plurielle, $\mathrm{n}^{\circ} 1$, p. 81-92;2010, "L'impact des violences conjugales sur les enfants. Pluralité d'interprétations d'une catégorie en formation », in Francequin G. (dir.) Tu me fais peur quand tu cries! Sortir des violences conjugales, Paris, Érès, p. 319-339.

- Jaspard M. et équipe Enveff, 2003, Les violences envers les femmes en France. Une enquête nationale, Paris, La Documentation française, coll. «Droits des femmes».

- Lagroye J. (dir.), 2003, La politisation, Paris, Belin, coll. "Socio-histoires».

- Lagroye J., François B. et Sawicki F., 2006, Sociologie politique, Presses de Sciences Po - Dalloz, (5 éd.), coll. " Amphi ».

- Lagroye J. et Offerlé M. (dir.), 2011, Sociologie de l'institution, Paris, Belin, coll. "Sociologiquement».

- Mathieu L., 2002, "La "conscientisation" dans le militantisme des années 1970 ", in Hamman P. et al. (dir.), Discours savants, discours militants : mélange des genres, Paris, L'Harmattan, coll. « Logiques politiques », p. 251270.

- Pagis J., 2009, "Les incidences biographiques du militantisme en mai 68. Une enquête sur deux générations familiales : des "soixante-huitards " et leurs enfants scolarisés dans deux écoles expérimentales (Vitruve et Ange Guépin) ", thèse de doctorat de sociologie, École des hautes études en sciences sociales (EHESS), Paris. 
- Picq F., 1993, Libération des femmes, les années mouvement, Paris, Le Seuil, coll. "Essais ».

- Poggi D., 2010, "Estime de soi et citoyenneté », in Francequin G. (dir.), Tu me fais peur quand tu cries! Sortir des violences conjugales, Paris, Érès, p. 8389.

- Snow D.-A., Rochford Jr B., Worden S.-K. et Benford R.-D., 1986, « Frame alignment processes, micromobilization, and movement participation ", American Sociological Review, vol. 51, n 4, p. 464-481. 